\title{
Solution of the SIR Mathematical Model for the Spread of Covid-19 Using GeoGebra
}

\author{
Marcellinus A. Rudhito ${ }^{1, *}$ Dewa P. W. Putra ${ }^{2}$ \\ 1,2Department of Mathematics Education, Universitas Sanata Dharma, Yogyakarta \\ *Corresponding author. Email: rudhito@usd.ac.id
}

\begin{abstract}
This study aims to find solution of the SIR modelling for the spread of Covid-19 in populations of an area for normal, new normal and lockdown conditions using GeoGebra. This research consists of two stages of numerical assessment of mathematical model completion using GeoGebra spreadsheets and building GeoGebra Applets for system simulations. The results show that the SIR model for the spread of Covid-19 can be solved numerically by using GeoGebra spreadsheets, by first changing the system of differential equations into systems of difference equations. Furthermore, by using existing facilities in GeoGebra, GeoGebra Applets can be arranged to simulate system dynamics for normal, new normal and lockdown conditions.
\end{abstract}

Keywords: SIR, Mathematical Model, Covid-19, GeoGebra.

\section{INTRODUCTION}

Experts modeling the spread of the disease, have started to study Covid-19. One of the studies made is a mathematical modeling of the spread of Covid-19. Lin [1] and Rong [2] investigated of the spread of Covid-19 in Wuhan City using the SEIR (Susceptible-ExposedInfectious-Removed) Model. The mathematical concept used in this model is a differential equation. The mathematical concept used in Covid-19 modeling usually uses advanced mathematical concepts, such as differential equations, time series analysis. The construction of the model and its solutions are technically difficult to understand for the general public. The models and solutions provided are not fully understood by the community. Assumptions in the model after being converted to parameters make it more difficult for the general public to understand the model. Therefore, people need to be given a simple understanding of the concept of mathematical models used for the spread of Covid-19, for example assumptions, modeling, completion, simulation results and predictions.

Solution and simulation of mathematical models become an important part. The results of this simulation can show visually about the current situation and predictions of a situation. Software that can be used to make simulations of a mathematical model of the spread of diseases including, MATLAB, Mathematica, GeoGebra, and others. GeoGebra is a software that provides facilities for solving equations (polynomial, exponent, differential) with graphical and numerical approaches. The solution of certain mathematical models can be solved using GeoGebra and provides a visual representation. Model simulation activities using GeoGebra can also be designed interactively, i.e. there is a slider facility that can be used to design parameters in mathematical models. Activities that can take place interactively are expected to make it easier to do or understand the results of simulations of the mathematical model for the spread of Covid-19.

There are at least five types of disease spread modeling, i.e. compartment model, stochastic model, Phenomenological model or time growth curve model, "time series" or "predictive class of data mining and AI (Artificial intelligence)", and class hybrid models (a combination of two or more model classes). In this article, we will discuss the most widely known model, i.e. the compartment model, by taking the simplest model, the SIR model. This model is applied to three different regional conditions namely normal conditions (without intervention), new normal conditions (by applying, clean living patterns, maintaining physical distance) and lockdown conditions (area closure). Next, settlement and simulation will be arranged with GeoGebra Applets so that the simulation the model can be done interactively and can be well understood by the general public. The novelty of this research is to determine SIR modeling 
solutions for Covid-19 disease using GeoGebra, create simulation applets and compare the results with existing analytical solutions.

\section{RESEARCH METHODOLOGY}

This research consists of two stages namely studying numerical completion of a SIR mathematical model and completing it using a spreadsheets and building an Applet for system simulation. Model simulation will use the GeoGebra Program. GeoGebra Applets are used for model construction simulations. Facilities in GeoGebra for simulation display, including Input bar (algebraic display), Bar graph (visual display), and Spreadsheet (numeric display). The parameters in the model will be displayed using a slider so that it is more interactive.

\section{RESULT AND DISCUSSION}

The SIR model is one model to describe the spread of an infectious disease that was first developed by Kermack and McKendrick (1926-1927). This model divides a study population $(N)$ into 3 compartments [3]. Each compartment in the population will be expressed in terms of time function. The function $S(t)$ states the number of individuals in the population who have the potential to be infected, ie individuals who have not been infected at the time $t$. The function $I(t)$ states the number of individuals in the population infected at the time $t$ and potentially transmits it to other individuals. Finally, the function $R(t)$ states the number of individuals who have been infected at a time $t$ and can be removed from the possibility of re-infection or can transmit it again to other individuals. Covid-19 is an infectious disease that can cause death for infected individuals. Therefore, in this article we used the SIR model by taking into account the mortality rate caused by the outbreak. This resulted in a decrease in the total population after the death due to the outbreak. This means that the total population is a function of decline with time units $N(t)$.

This model will use 3 parameters, namely the population death rate caused by the outbreak $(d)$, the recovery rate denoted by $\gamma$, and the contact rate of each infected $(I)$ individual with the susceptible infected person $(S)$ notated with $a$. This parameter $a$ will affect the transmission rate. Based on [3], the transmission rate is expressed in the following equation:

$$
\beta(N)=a N^{-0.95} \text {. }
$$

This SIR model is stated in the following system of differential equations

$$
\left\{\begin{array}{l}
S^{\prime}=-\beta(N) S I \\
I^{\prime}=\beta(N) S I-(\gamma+d) I \\
R^{\prime}=\gamma I \\
N^{\prime}=-d I
\end{array} .\right.
$$

This model will be solved using GeoGebra. The first step in completing this model is by changing each equation in the system above into a different equation (difference equation) [4]. Obtained a system of different equations as follows.

$$
\left\{\begin{array}{l}
\Delta S=-\beta(N) S I \Delta t \\
\Delta I=[\beta(N) S I-(\gamma+d) I] \Delta t \\
\Delta R=\gamma I \Delta t \\
N^{\prime}=-d I \Delta t
\end{array}\right.
$$

Next, model the solution at the time $t=t_{k}$, that is $S\left(t_{k}\right)=S\left(t_{k-1}\right)+\Delta S, I\left(t_{k}\right)=I\left(t_{k-1}\right)+\Delta I, R\left(t_{k}\right)=$ $R\left(t_{k-1}\right)+\Delta R, N\left(t_{k}\right)=N\left(t_{k-1}\right)+\Delta N$.

The next step is to iterate over the spreadsheets available on GeoGebra. Iteration is done to see the condition of each compartment in a certain time interval. The parameters in the model are estimated in value so that the iteration process can be carried out. Death rates by epidemics and cure rates were estimated using data on death rates by Covid-19 in Indonesia (https://kawalcovid19.id/) during March - June 2020. Parameter values $d$ are at intervals $0.02 \leq d \leq 0.09$ and parameter values are at intervals. Reproductive Number Covid-19 from 1 January 2020 to 7 February 2020 is at an interval $1.4 \leq \gamma \leq 6.49$ [5]. Reproductive Number is used to estimate the value of the contact rate parameter. Based on [3] and [6] the reproductive number value can be determined as follows

$$
\mathfrak{R}_{0}=\frac{N_{0} \beta\left(N_{0}\right)}{\gamma+d} \text {. }
$$

Based on equations (1) and (4) the parameter values $a$ are obtained at interval $0.0443 \leq a \leq 2.2181$. The interval values for the three parameters are created using the slider facility available in the GeoGebra menu. Suppose the initial value of each compartment is $N_{0}=$ $100001, S_{0}=10000, I_{0}=1$, dan $R_{0}=10000$. Figure 1 below is a picture of a spreadsheet display in the iteration process.

The next step is to visualize the solution of the model in Graph view. In the GeoGebra graphical display we plot each point $\left(t_{k}, N\left(t_{k}\right)\right),\left(t_{k}, S\left(t_{k}\right)\right),\left(t_{k}, I\left(t_{k}\right)\right)$ and $\left(t_{k}, R\left(t_{k}\right)\right)$ with $k=0,1,2,3, \ldots$. The final step is to connect each two consecutive dots in each compartment with a line segment.

\subsection{Simulation for Normal Condition}

A situation without intervention means that each individual in the entire population has no limited interactions with other individuals. This causes the value of the contact rate parameter $a$ to be fixed at all times. From the contact rate parameter which is in the interval $[0.0433,2.2181]$, for the simulation example, we take the mean value of the interval, which is $a=1.1312$. Furthermore, by using parameter values $d=0.052$ and 


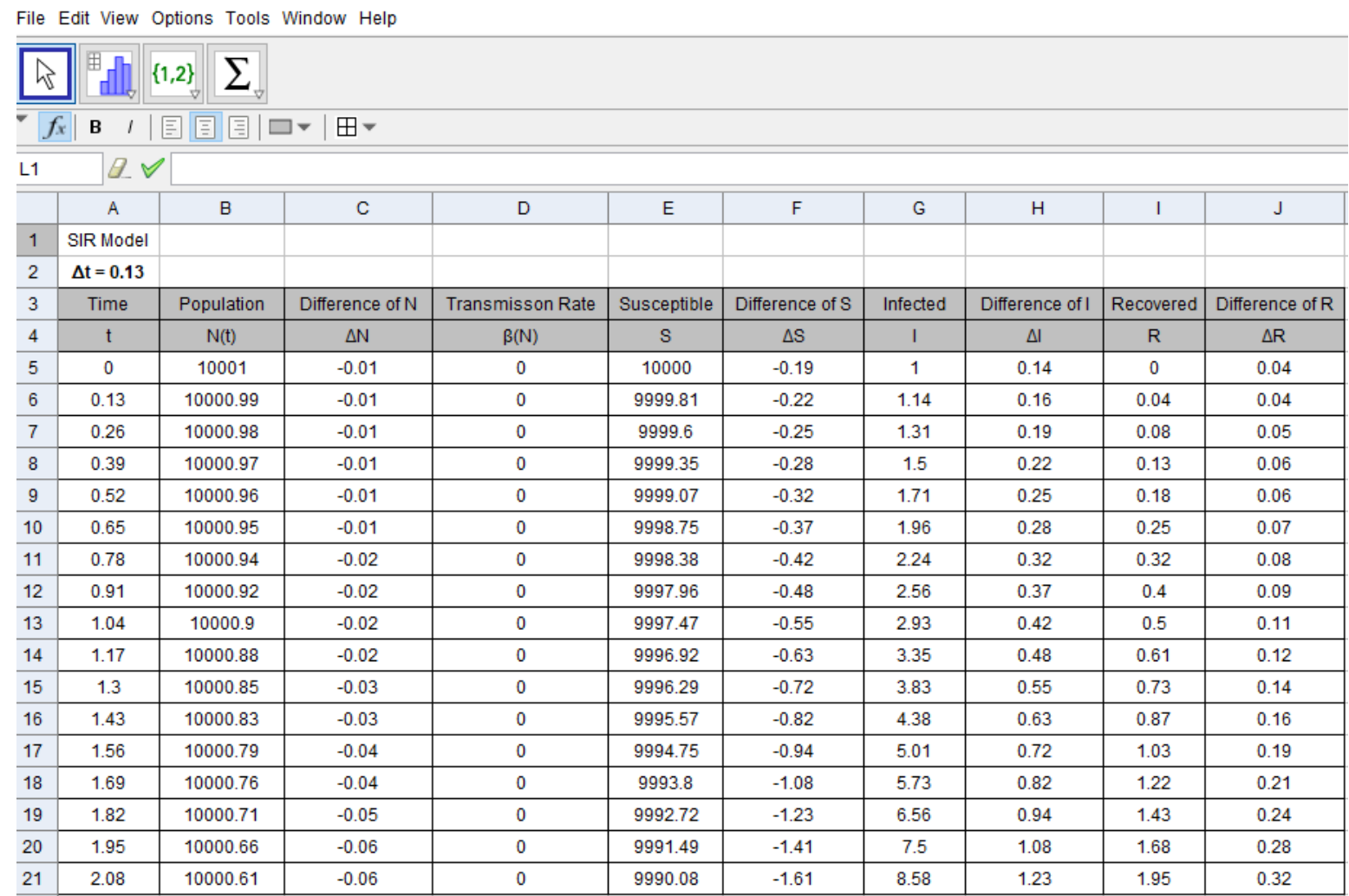

Figure 1 Iteration with spreadsheets in GeoGebra

$\gamma=0.35$ obtained model solutions using GeoGebra in the following Figure 2.

Based on Figure 2, the peak of the pandemic occurred in the ninth week with a total of 4579 infected individuals from 100001 individuals in the initial population. As of the 26 th week there were 1,284 deaths.

\subsection{Simulation for New Normal Condition}

This situation provides restrictions on the interaction of individuals in the population. Limitation of interaction is done by limiting social distance. In addition, interventions were also given to individual habits, namely by using masks, diligently washing hands and avoiding touching the nose, eyes and mouth using hands. These interventions will have an impact on the transmission rate of the virus. In this article, the intervention is expected to reduce the transmission rate by up to $50 \%$. The model solution in this situation can be seen in the following Figure 3.

In this model, a new intervention starts from 4th week. Parameter values are applied from the beginning up to before the 4th week and after that the parameter values. Based on this, information is obtained that the peak of the pandemic will occur in the 12th week with a total of 2079 infected individuals from a total of 100001 population. As of the 26th week, there were 7488 individuals recovering and 1112 deaths.

\subsection{Simulation for Lockdown Condition}

The Lockdown situation is a strategy to reduce the transmission rate to a minimum. Individual mobility is not permitted in this situation, so hope is not contact at all. In this model, lockdown is applied from the 4th week. The virus transmission rate is estimated to drop by $50 \%$ after 2 weeks of lockdown and then immediately drop to 0 after the 6 th week. This estimate was taken on the grounds that the virus incubation period is 2 weeks. So, for 2 weeks after lockdown there will still be infected individuals who come from the compartment. The model solution with an intervention in the form of a lockdown using GeoGebra can be seen in the following Figure 4 bellow.

Based on the solution of the model above, information is obtained that a pandemic peak will occur at week 6 with a total of 427 infected individuals out of 100001 the total population. Until the 26th week there were no more individuals infected with Covid-19. Based on the three strategies above, the following is summarized information regarding the spread of Covid19.

Based on the three conditions above, the following is summarized information regarding the spread of Covid19 in the following Table 1 bellow. 
File Edit View Options Tools Window Help

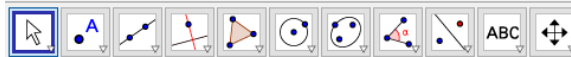

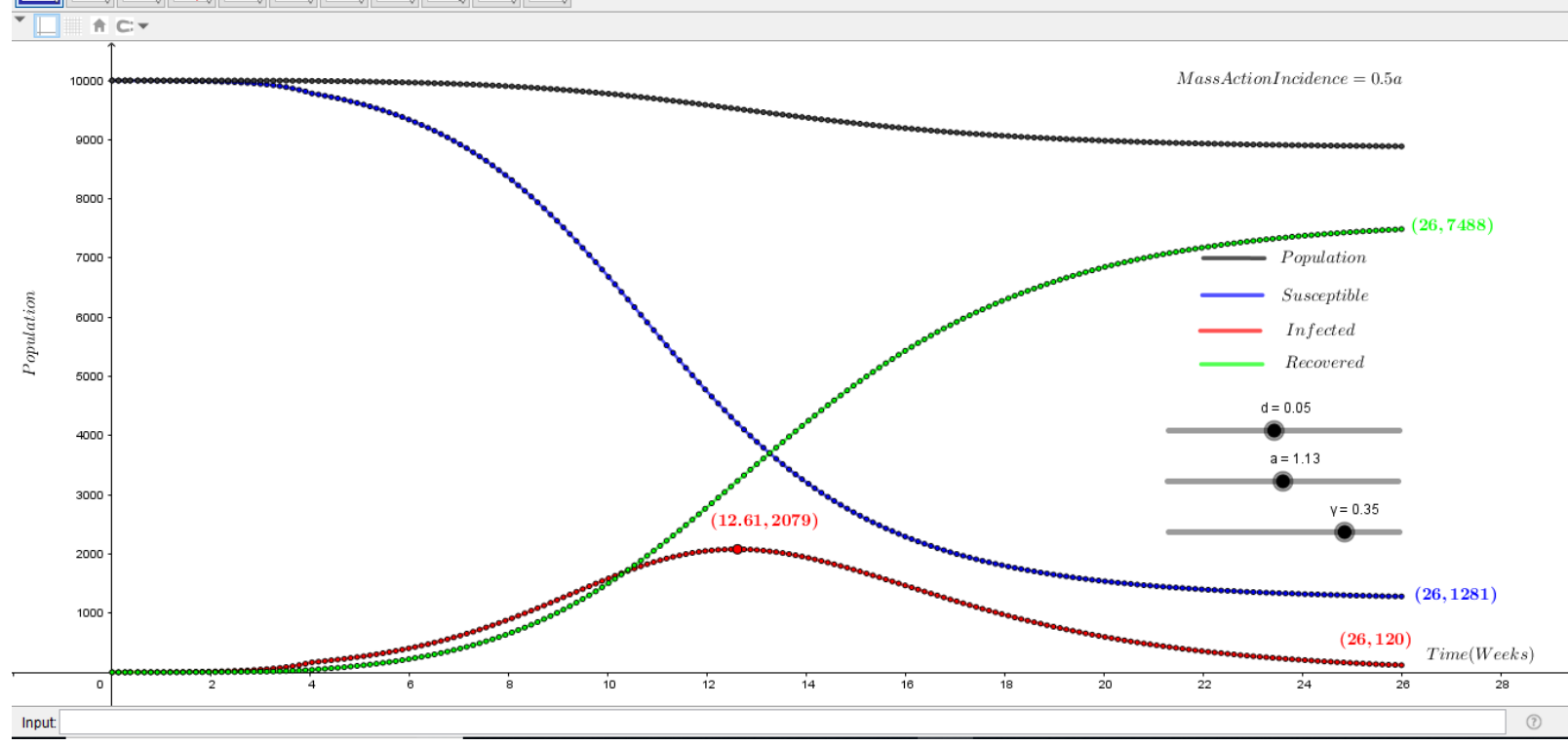

Figure 2 GeoGebra Applets for normal condition simulation

2 Model Diskrit SIR dengan kematian.ggb

File Edit View Options Tools Window Help

4. A File Edit View Options Tools Window Help

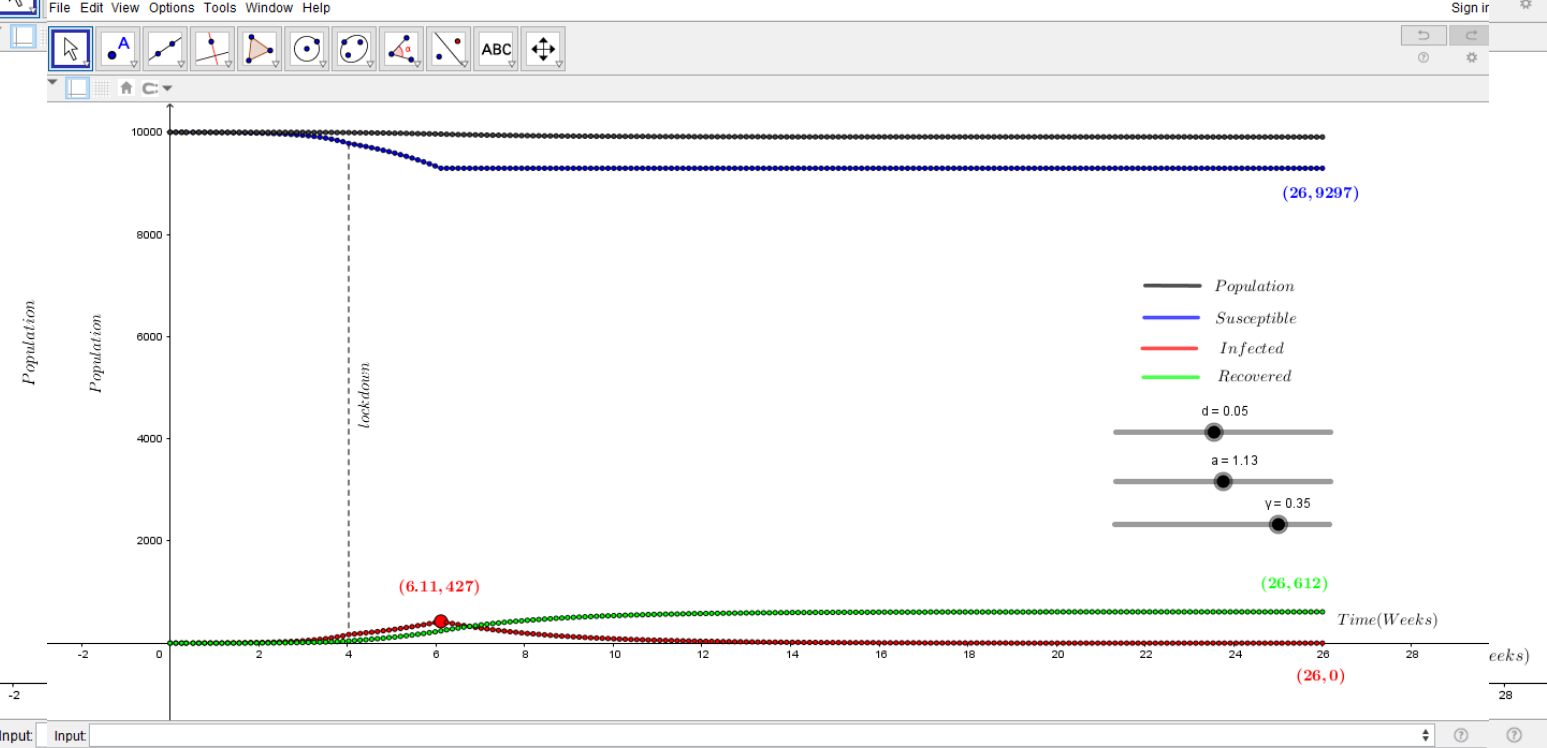

Figure 3 GeoGebra Applets for new normal condition simulation

Table 1 Comparison of simulation results for the three conditions

\begin{tabular}{|l|c|c|c|}
\hline \multicolumn{1}{|c|}{ Conditions } & Start (week) & Pandemic peak (weak) & Percentage of Infected \\
\hline Normal & - & 9 & $46 \%$ \\
\hline New Normal & 4 & 12 & $21 \%$ \\
\hline Lockdown & 4 & 6 & $4 \%$ \\
\hline
\end{tabular}




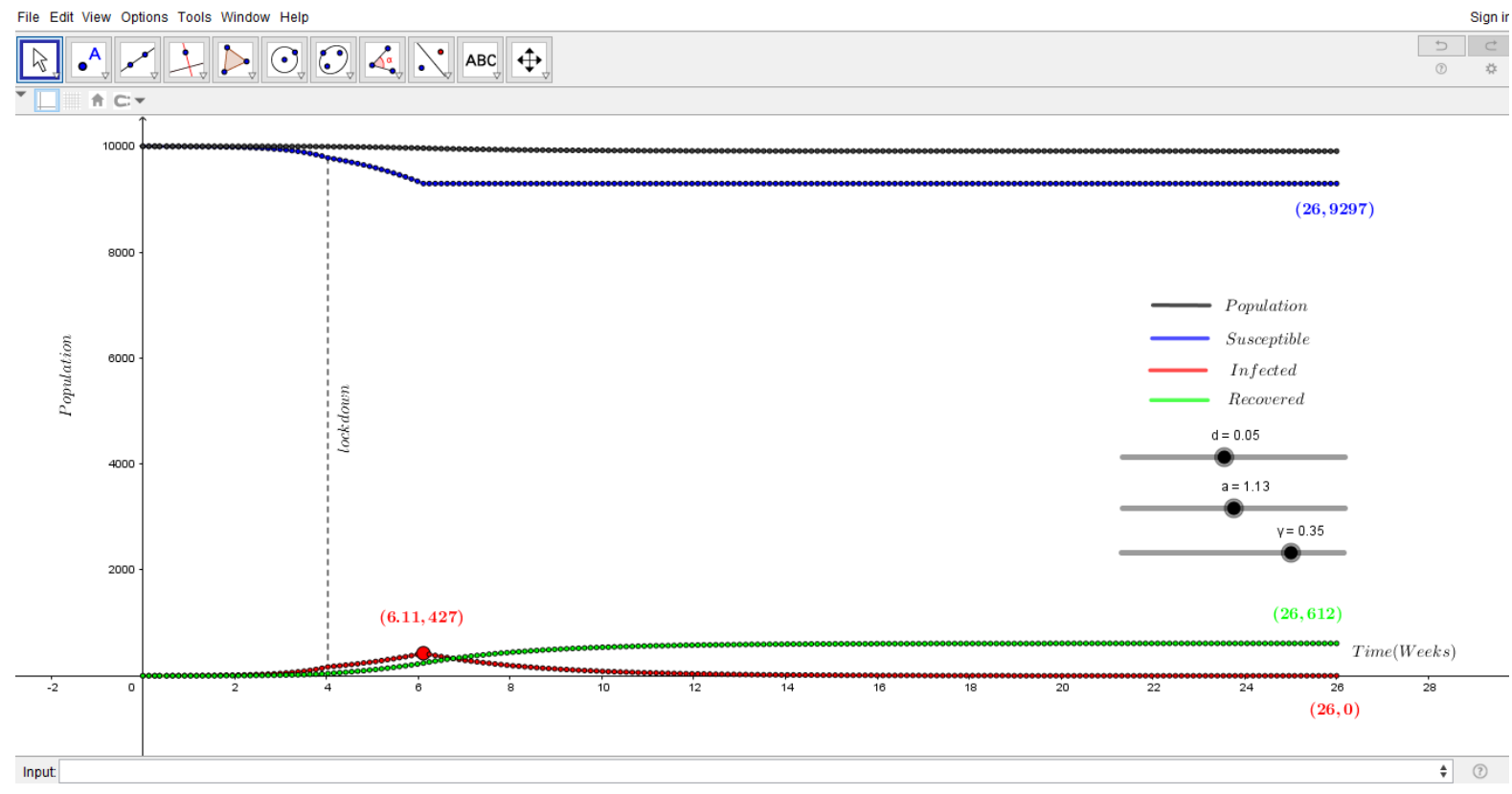

Figure 4 GeoGebra Applets for lockdown condition simulation

From the results of modeling solutions using GeoGebra above, it appears that GeoGebra can be used to determine solutions for modeling the spread of Covid-19 as is the case for flu epidemics in the source [4]. In creating GeoGebra applets to simulate modeling solutions, GeoGebra seems to be heavy on doing calculations in spreadsheets and less practical in copying formulas than Excel. But for graph view and display points in graph GeoGebra is still better than Excel.

The exact solution of the system of differential equations (2) is given by

$$
\begin{aligned}
& S(t)=S_{0} u(t) \\
& I(t)=\frac{\gamma}{\beta} \ln u(t)-S_{0} u(t)+N
\end{aligned}
$$

with $u(t)=e^{-\frac{\beta}{\gamma} R(t)}$ [7]. Based on these solutions, the comparison of numerical solutions and exact solutions in the Susceptible and Infected compartments is an average of $3 \%$ difference.

\section{CONCLUSION}

From the above discussion, it appears that we can solve the SIR model without going through the exact solution of the differential equation system with a fairly good level of accuracy. The SIR model for Covid-19 distribution can be solved numerically by using GeoGebra spreadsheets, by first changing the system of differential equations into systems of difference equations. The next step is to iterate over the spreadsheets available on GeoGebra. Iteration is done to see the condition of each compartment in a certain time interval. The final step is to describe the solution of the model in Graph view. Furthermore, by using existing facilities in
GeoGebra, GeoGebra Applets can be arranged to simulate the dynamics of the system for normal conditions, new normal and lockdown. The simulation with the GeoGebra Applets above has been quite heavy and it takes quite a long time to iterate, so for the next more complex system requires a more powerful spreadsheet program.

\section{ACKNOWLEDGMENTS}

We would like to thank the Sanata Dharma University Research Institute for providing support and research funding in the Covid-19 field.

\section{REFERENCES}

[1] Lin, Qianying, dkk. 2020. A Conceptual Model for the Coronavirus Disease 2019 (COVID 19) outbreak in Wuhan, China with Individual Reaction and Govermental Action, International Journal of Infectious Diseases, Vol. 93 pp. 211-216.

[2] Rong. Xinmiao, dkk. 2020. Effect of Delay in Diagnosis on Transimission of COVID-19. Mathematical Biosciences and Engineering. 17(3), pp. $2725-2740$

[3] Brauer, Fred, Carlos Castillo-Chavez, dan Zhilan Feng. 2019. Mathematical Models in Epidemology. New York : Springer Science+Bussinnes Media, LLC.

[4] Jonas Hall, Thomas Lingefjärd. 2017. Mathematical Modeling : Applications with GeoGebra. New Jersey : John Wiley \& Sons, Inc. 
[5] Liu, Ying et al. 2020. The Reproductive Number of COVID-19 is Higher Compared to SARS Coronavirus. International Journal of Travel Medicine. Pp 1-4. Doi : 10.1093/jtm/taaa021.

[6] van den Driessche P.,2017, Reproduction numbers of infectious disease models, Infectious Disease Modelling (2017), doi: 10.1016/j.idm.2017.06.002.

[7] Harko,T.,Lobo, F.S.N., and Mak, M.K. 2014. Exact Analytical Solutions of Susceptible-InfectedRecovered (SIR) Epidemic Mocel and of the SIR Model with Equal Death and Birth Rate. arXiv.1043.2160v1. 